\title{
Imaging Modalities for Focal Nodular Hyperplasia and Hepatocellular Adenoma
}

\author{
Jacomina W. van den Esschert ${ }^{a}$ Thomas M. van Gulik ${ }^{a}$ Saffire S.K.S. Phoa ${ }^{b}$ \\ Departments of a Surgery and ${ }^{b}$ Radiology, Academic Medical Center, University of Amsterdam, Amsterdam, \\ The Netherlands
}

\section{Key Words}

Liver cell adenoma · Focal nodular hyperplasia • Magnetic resonance imaging • Ultrasound $\cdot$ Spiral computed tomography $\cdot$ Radionuclide imaging

\begin{abstract}
Background/Aims: There are several imaging modalities available for the detection of focal liver lesions. Differentiation between focal nodular hyperplasia (FNH) and hepatocellular adenoma (HCA) is important because of the consequences for management. However, differentiation based on imaging alone still shows limitations. Methods: We reviewed the literature for typical features of FNH and HCA on radiologic and nuclear imaging with emphasis on differentiation of both lesions. Results: Seven articles describe the performance of an imaging modality for the differentiation between FNH and HCA. Limitations of these studies are the small sample size and/or the lack of comparison with the 'gold standard', i.e. histological diagnosis. No studies are available that compare the accuracy of several imaging modalities in the differentiation of FNH and HCA. Conventional ultrasound (US) is not useful in the differentiation because of the non-specific features. On contrast-enhanced US, the arterial filling direction of $\mathrm{FNH}$ is centrifugal and centripetal in case of HCA. The parenchymal enhancement of FNH is sustained in the portal venous and delayed phases, but shows
\end{abstract}

rapid washout in case of $\mathrm{HCA}$. Multiphase $\mathrm{CT}$ scan can differentiate FNH from HCA when there is a central scar. FNH may have a slightly higher relative enhancement in the arterial phase. On MRI with hepatocyte-specific contrast agents, HCA does not show contrast uptake in the hepatobiliary phase in contrast to $\mathrm{FNH}$. Conclusion: We conclude that there is limited evidence of the diagnostic performance of currently used imaging modalities for the differentiation of FNH and HCA. We therefore propose a prospective study (DiFA trial) to determine the accuracy of several radiologic and nuclear imaging studies in differentiating FNH and HCA.

Copyright $\odot 2010$ S. Karger AG, Base

\section{Introduction}

Due to the increased use of abdominal imaging, benign liver tumors are detected as an incidental finding more frequently. Focal nodular hyperplasia (FNH) and hepatocellular adenoma (HCA) are both benign lesions which predominantly occur in young and middle-aged women. Differentiation of FNH from HCA is important because of the consequences for management of both these lesions. HCA carries a risk of spontaneous bleeding and malignant transformation as pointed out elsewhere in this issue $[1,2]$.

\section{KARGER}

Fax +4161306 1234

E-Mail karger@karger.ch

www.karger.com (c) 2010 S. Karger AG, Basel

0253-4886/10/0271-0046\$26.00/0

Accessible online at:

www.karger.com/dsu
S.S.K.S. Phoa, MD, PhD

Department of Radiology, Academic Medical Center

Meibergdreef 9, NL-1105 AZ Amsterdam (The Netherlands)

Tel. +31 20566 9111, E-Mail s.s.phoa@amc.uva.nl 
Differentiation may be difficult based on imaging studies alone, because of the radiological similarities of both tumors. Biopsy and histological assessment of the tumor for a definitive diagnosis of FNH or HCA is mandatory in such cases [3]. In recent years many new imaging modalities have been introduced, e.g. MRI with hepatobiliary contrast agents and contrast-enhanced ultrasound (US). The question arises which imaging modality performs best in differentiating FNH from HCA, and whether we can rely on imaging for diagnosis rather than to obtain histopathological confirmation.

We reviewed the literature for studies that described the typical features of FNH and HCA on several imaging modalities, and for studies that focused on the ability of imaging modalities in differentiating FNH from HCA.

\section{Methods}

The literature was reviewed for typical features of FNH and HCA on radiologic and nuclear imaging (US, multiphase CT, MRI and nuclear imaging) with emphasis on differentiation of both lesions. A literature search was performed using the Medline database. The applied search terms were a combination of the $\mathrm{MeSH}$ terms 'focal nodular hyperplasia', 'liver cell adenoma', 'magnetic resonance imaging', 'ultrasound', 'spiral computed tomography' and 'radionuclide imaging' with addition of the word 'differentiation'. The search was limited to English language. Additional articles were reviewed from the bibliography of articles cited.

\section{Results}

Of the 55 hits, 7 studies specifically assessed the use of an imaging modality in the differentiation of FNH and HCA. These studies are discussed below.

\section{Ultrasound}

Both FNH and HCA show non-specific features on gray-scale US. FNH is usually slightly hypo- or isoechoic and very rarely hyperechoic. Sometimes the lesion is only detected by visualization of a pseudocapsule which is due to compression of the surrounding liver tissue or vessels. A central scar and septae are typical findings of FNH, but more than half of the FNH are devoid of a central scar [4-8]. HCA is also hypoechoic in the majority of cases. However, HCA may appear hyperechoic because of abundant fat, fibrosis, or bleeding inside HCA. Calcifications may be present and show up as hyperechoic foci with acoustic shadowing $[9,10]$.

Imaging of FNH and HCA
Color Doppler US is able to show peri- and intratumoral vessels [10-12]. Bartolozzi et al. [13] performed a Doppler US study in which they compared power Doppler and color Doppler sonography in 29 patients with 22 histologically proven FNHs and 9 HCAs. FNH was characterized by the presence of multiple well-defined vessels with a pulsatile Doppler spectrum radiating from the center to the periphery of the lesion. This finding was present in $91 \%$ of the lesions on power Doppler and in $68 \%$ on color Doppler sonography. In contrast, HCA showed vessels running along the periphery of the lesion associated with multiple vascular pedicles in the central portion with a venous Doppler spectrum, in 89 and $67 \%$ on power Doppler and color Doppler sonography, respectively. Power Doppler imaging appeared to be superior to color US in the depiction of those intratumoral flow characteristics.

To improve the characterization of liver lesions on US, contrast agents consisting of microbubbles have been developed. Nowadays, second-generation contrast agents (e.g. Sonovue; Bracco Imaging, Milan, Italy) and continuous (real-time) US scanning are often used. Burns and Wilson [14] assessed the concordance of enhancement patterns of focal liver lesions on contrast-enhanced US with patterns on contrast-enhanced CT and MRI. They concluded that US showed a high concordance with arterial phase CT or MRI. This concordance was much less for the portal phase, probably due to lack of diffusion of the microbubbles into the interstitium.

After contrast administration, FNH typically shows centrifugal filling preceded by a spoke-wheel pattern enhancement in the early arterial phase. This spoke-wheel pattern is less present in lesions $<3 \mathrm{~cm}$. During the portal venous and delayed phases, FNH appears hyper- or isoechoic in comparison with surrounding liver parenchyma. The presence of a non-enhancing central scar was found to be a distinctive feature of FNH [15-23]. HCA shows subcapsular feeding arteries with mixed or centripetal filling. The clear enhancement in the arterial phase becomes less intense in the portal venous and delayed phases [24].

Dietrich et al. [25] performed a study in 32 patients to differentiate FNH from HCA with contrast-enhanced US. All lesions showed pronounced enhancement in the arterial phase. In the portal phase, $96 \%$ of patients with FNH showed sustained enhancement. In contrast, all patients with HCA showed rapid washout of the contrast leading to no enhancement during the portal venous phase. Kim et al. [26] performed a retrospective study to determine the differential features of FNH and HCA on 
contrast-enhanced US. Their conclusion was that the features in the early arterial phase contributed most to the differentiation of FNH and HCA by showing the arterial phase filling direction and arterial morphology. FNH showed centrifugal filling (74-91\%) and stellate vascularity (60-67\%), whereas HCA was less reliably predicted by centripetal or mixed filling (86\%) without stellate vascularity (11-16\%). They concluded that sustained portal enhancement was more common in FNH (86-91\%) than in HCA (47-63\%).

In short, conventional US is not useful in differentiating FNH from HCA because of the non-specific features, except for a central scar. On Doppler US and contrastenhanced US, FNH typically shows centrifugal, arterial vascularity and HCA typically shows centripetal vascularity with a flat continuous Doppler spectrum. The additional value of real-time US with microbubble contrast lies in the enhancement pattern of the liver parenchyma. Both lesions show pronounced enhancement in the arterial phase. In case of $\mathrm{FNH}$, parenchymal enhancement sustains in the portal venous and delayed phases, but in case of HCA washes out rapidly.

\section{Multiphase CT Scan}

Many studies assessed the use of multiphase CT scan for the detection of focal liver lesions, including FNH and HCA. On plain CT scan, FNH is usually homogeneous and iso- or slightly hypointense compared to normal liver tissue. In case of steatosis of the liver parenchyma, FNH may appear hyperintense due to the low attenuating fatty liver $[27,28]$. Calcifications are rarely seen $[27,29$, 30]. HCA may show a variety of density patterns on plain CT scan due to fat, necrosis or hemorrhage. Calcifications are more common than in $\mathrm{FNH}$ and are present in $5-15 \%$ of the lesions [31].

After contrast administration, rapid homogeneous enhancement of well-delineated, lobular-shaped FNH is seen in the arterial phase with a hypodense central scar in some cases. An enlarged feeding artery is often visible. In the portal venous and delayed phase, the FNH becomes isodense and may be difficult to detect. The central scar and septations typically show late enhancement due to diffusion of the contrast material into the stroma of the lesion [4, 27, 28, 30,32-37]. A central scar is seen on CT in approximately $50 \%$ of the lesions. Large FNHs $(>3 \mathrm{~cm})$ are significantly more likely to reveal central scarring, vascular displacement and abnormal vessels around the lesions than smaller lesions [27, 38]. A pseudocapsule is seen in $8 \%$ of the FNHs [27]. A well-circumscribed HCA also becomes hyperdense in the early arte- rial phase and slightly hyper- to isodense in the portal phase. Peripheral enhancement may be seen reflecting the presence of the large subcapsular feeding vessels [10]. On delayed phase imaging, a washout phenomenon may occur when contrast washout is rapid, rendering the lesion hypodense in comparison to the surrounding liver tissue. A pseudocapsule is present in $25-30 \%$ of the HCAs $[10,31]$.

Ruppert et al. [36] performed a study in 45 histologically proven FNHs and 27 HCAs to evaluate the use of triphasic CT imaging for differentiating FNH and HCA. They found a central scar in $65 \%$ of all FNHs $(29 \%$ in $\mathrm{FNH}<3 \mathrm{~cm}$ and $82 \%$ in $\mathrm{FNH}>3 \mathrm{~cm}$ ) and none in case of a HCA. Subcapsular arteries were detected in none of the FNHs and in 39\% of the HCAs. In addition, they quantified dynamic enhancement patterns. FNH enhanced more homogeneously and arterial enhancement was stronger than in HCA. HCA showed more washout in the portal phase; $22 \%$ of the HCAs were hypodense, whereas FNH remained iso- or hyperdense. They concluded that FNH can reliably be diagnosed if relative enhancement is $>1.6$ in the arterial phase.

In conclusion, multiphase CT scan can differentiate FNH from HCA based on the presence of a central scar, the slightly higher relative enhancement in the arterial phase and the presence of central feeding arteries. However, these findings are not always present or visible on CT (fig. 1, 2). HCA shows heterogeneous densities on plain CT due to fat, necrosis, hemorrhage or calcifications and a washout phenomenon may occur.

\section{MRI Scan}

Owing to high soft-tissue contrast of MRI, FNH and HCA can be depicted on non-enhanced MRI. Almost all FNH lesions are slightly hyper- or isointense or not visible on $\mathrm{T}_{2}$ images of unenhanced MRI. A central scar, typically hyperintense, may be seen in approximately $30 \%$ of the lesions, mostly in the lesions $>3 \mathrm{~cm}$. On the $\mathrm{T}_{1} \mathrm{im}$ ages, most lesions are iso- or slightly hypointense while the central scar appears hypointense [39-48]. HCA is also mainly slightly hyper- to isointense on $\mathrm{T}_{2}$ images. On $\mathrm{T}_{1}$ images, all possible signal intensities are mentioned in the literature. For example, Grazioli et al. [41] showed that HCA was iso- or slightly hypointense in $74 \%$ and slightly hyperintense in $20 \%$ of the lesions. Chung et al. [49] also showed that the minority of the lesions were hyperintense (35\%), while according to Paulson et al. [50] and Arrive et al. [51], HCA appeared hyperintense in 51 and $59 \%$ of the cases, respectively. HCA may show a more heterogeneous signal intensity than FNH due to hemorrhage, fat or ne- 
Fig. 1. Multiphase CT scan of a typical FNH. On plain CT: a slightly hypodense lesion is visible in liver segment 4 (a). In the arterial phase, the hypervascular lesion appears hyperdense with a hypodense central scar (b). In the portal venous phase, the FNH becomes iso- to hypodense and the central scar is still hypodense (c). In the equilibrium phase, an isodense lesion is seen. The central scar shows contrast enhancement and is therefore poorly recognized (d).

Fig. 2. Multiphase CT scan of an atypical FNH without a central scar. On the arterial phase images, homogeneous enhancement of the lesion $(>3 \mathrm{~cm})$ is seen without a central scar (a). The FNH sustains enhancement in the portal venous (b) and equilibrium phase (c) without signs of a central scar.
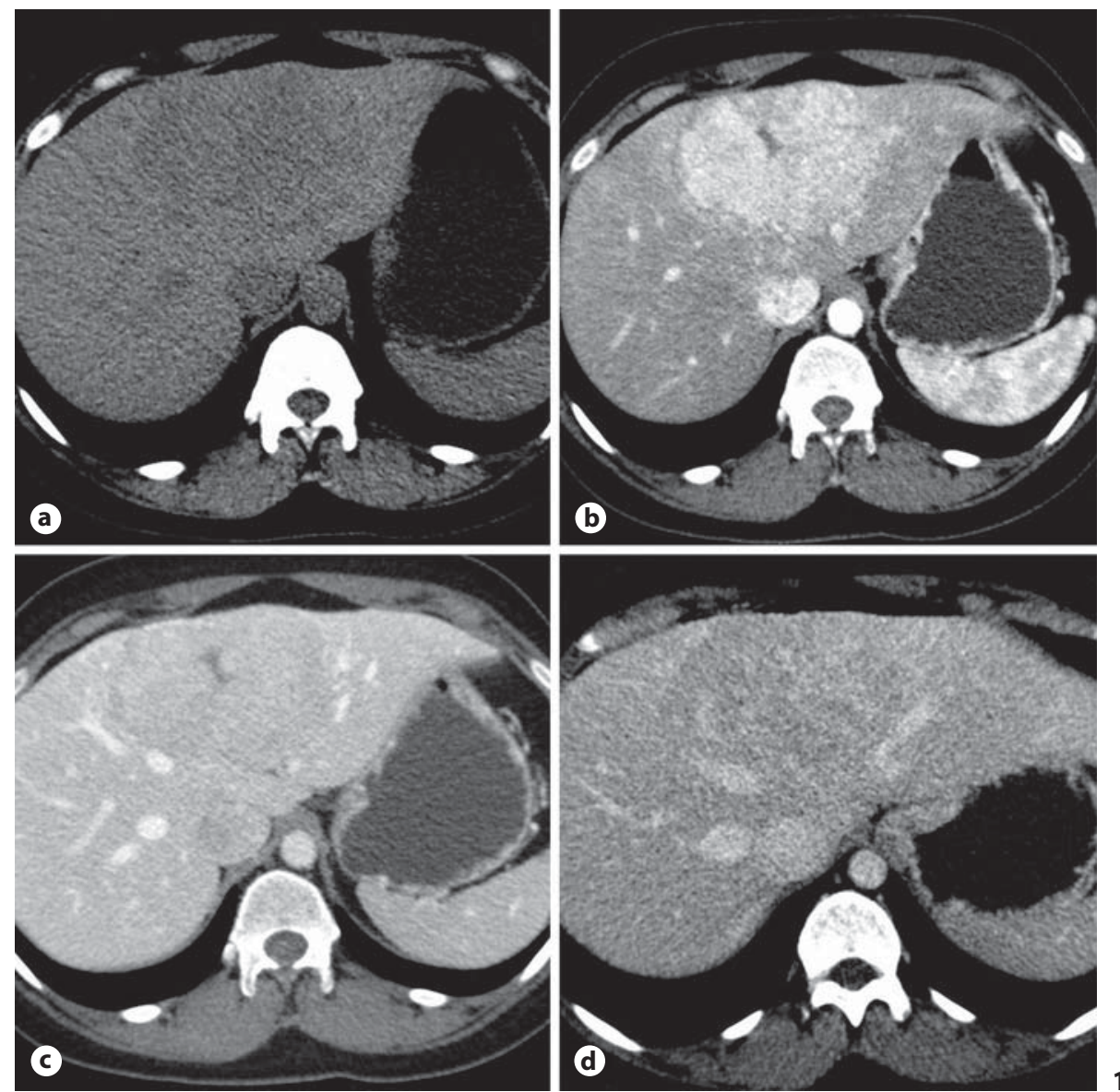

1
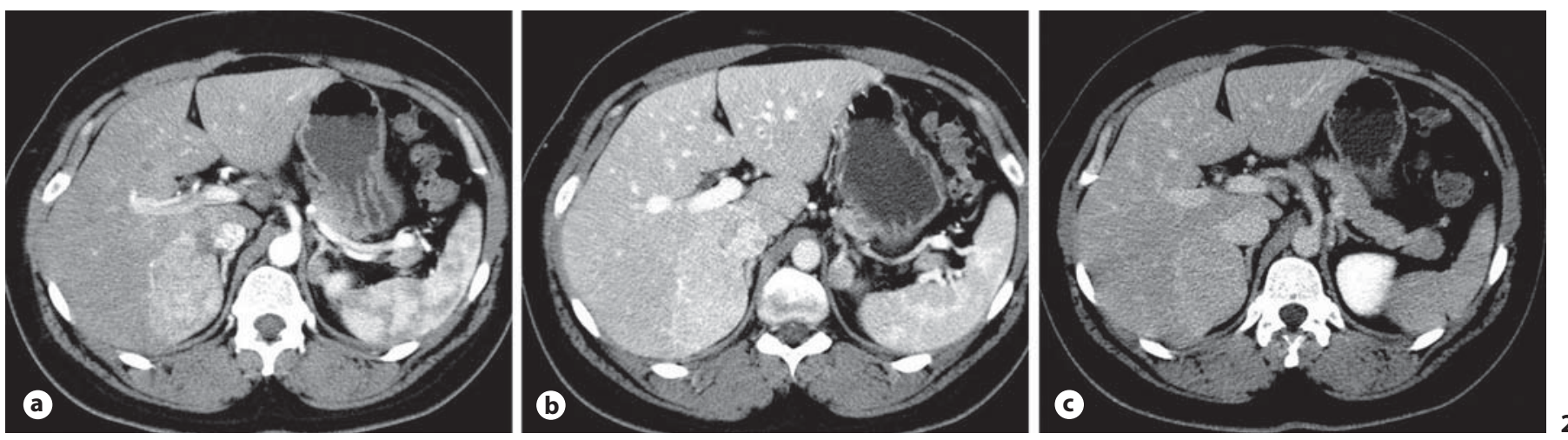

crosis. MRI is superior to CT in detecting small amounts of fat in the liver or in a focal liver lesion, using in- and out-of-phase imaging sequences. Old bleeding in the lesion may cause a scar-like appearance (fig. 3).

There are several liver-specific contrast agents available for MRI. Superparamagnetic iron oxide (SPIO) and USPIO (ultrasmall SPIO) particles are taken up by the re- ticuloendothelial system. Uptake by Kupffer cells will lead to a loss of signal of the liver on $\mathrm{T}_{2}$-weighted MR images $[47,53,54]$. The iron oxide particles are used to better detect lesions in the liver that do not contain Kupffer cells, e.g. metastasis. In FNH the amount of functional Kupffer cells is higher than in HCA. Therefore, FNH demonstrates more signal loss on MRI after ferumoxide 

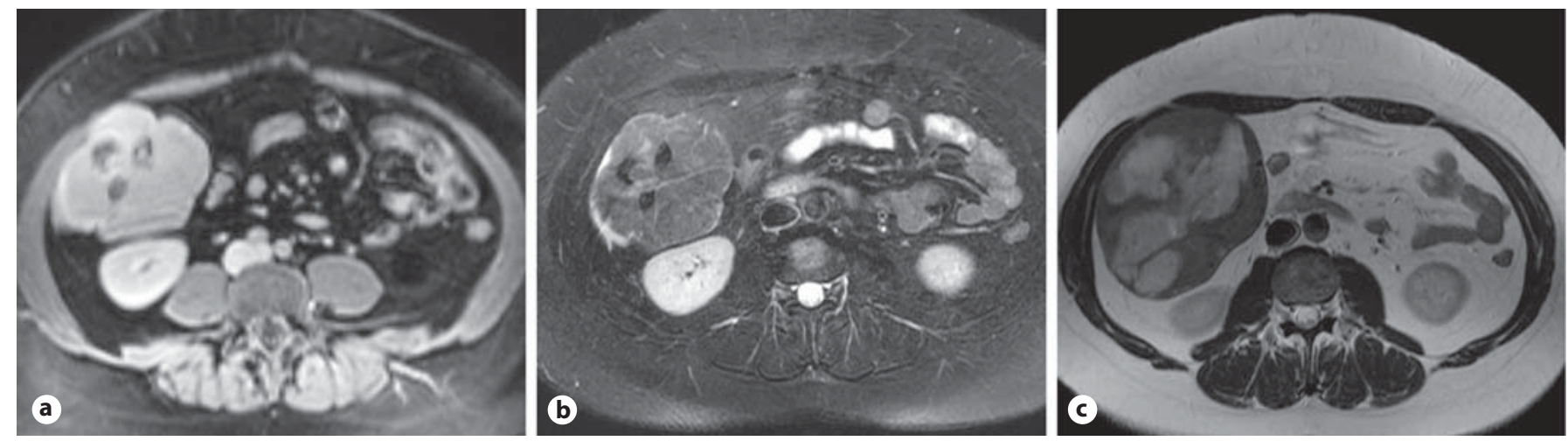

Fig. 3. MRI adenoma with old central bleeding. On the $\mathrm{T}_{1}$-weighted image (fat sat) in the arterial phase, strong enhancement of the lesion is seen except for the central area (a). On $\mathrm{T}_{2}$-weighted $\mathrm{MR}$ image the central scar shows a low signal which is not consistent with a scar in $\mathrm{FNH}(\mathbf{b})$. An unenhanced $\mathrm{T}_{1}$-weighted $\mathrm{MR}$ image of the same patient 3 months earlier shows a large central area with high signal which is consistent with an acute bleeding. At that time, the patient did not have acute symptoms (c). compared to HCA. However, the diagnosis of FNH cannot reliably be made on the basis of ferumoxide as contrast agent $[39,55,56]$.

Mangafodipir trisodium (Teslascan; Amersham Health, Oslo, Norway) is a MR contrast agent that is selectively taken up by hepatocytes, leading to increased signal intensity on $\mathrm{T}_{1}$-weighted images in normal liver parenchyma. It is excreted via the biliary system. With this contrast agent, hepatocyte-containing liver tumors can be differentiated from tumors of non-hepatocyte origin, e.g. metastases [55, 57].

Gadobenate dimeglumine (Gd-BOPTA, Multihance; Bracco Imaging) and gadoxetic acid (Gd-EOB-DTPA, Primovist; Schering) are both contrast media with combined perfusion and hepatocyte-selective properties. Firstly, these contrast media are visible during the vascular phase due to the non-specific gadolinium chelates. Secondly, they are partly taken up by hepatocytes and excreted into the bile ducts [58]. The latter property is of importance in the differentiation of FNH and HCA, because HCA does not contain bile ducts in contrast to FNH (fig. 4, 5).

Grazioli et al. [41] performed a prospective, multicenter study to differentiate FNH from HCA with MRI and Gd-BOPTA in 73 patients with FNH, 27 with HCA, and 8 with adenomatosis. On $\mathrm{T}_{1}$-weighted images, $95 \%$ of the FNHs appeared homogeneously hyperintense in the arterial phase. In the portal venous and equilibrium phases, the FNHs remained slightly hyperintense (47 and $33 \%$, respectively) or became isointense compared to the surrounding liver parenchyma. The central scar was mainly seen as a hypointense feature in the arterial phase and became hyperintense in the equilibrium phase. The enhancement pattern of HCA was similar to that of FNH after contrast administration, i.e. hyperintense in the arterial phase (96\%) and hyper- to isointense in the portal venous and equilibrium phases. In the hepatobiliary phase, when contrast is taken up by the hepatocytes and excreted via the bile ducts, FNH appeared hyperintense $(68 \%)$ or isointense (29\%). In contrast, all HCAs appeared hypointense in this delayed phase.

Recently, Zech et al. [48] performed a prospective study to evaluate the diagnostic performance of MRI with Primovist in comparison to pre-contrast MRI and dynamic CT in the diagnosis of FNH. They concluded that MRI with Primovist was superior to pre-contrast MRI alone and to dynamic CT. However, mixed uptake in FNH still resulted in misdiagnosis of FNH for HCA in 25 of 59 lesions by 1 of the 3 blinded radiologists.

To sum up, MRI with hepatocyte-specific contrast agents performs better in diagnosing FNH than multiphase CT scan. The typical enhancement patterns of both FNH and HCA are similar in the arterial and portal venous phase. In contrast to FNH, HCA does not show contrast uptake in the hepatobiliary phase. However, contrast uptake in FNH may show a mixed response making characterization difficult (fig. 6). The central scar in FNH is typically hyperintense on $\mathrm{T}_{2}$-weighted images and is mainly seen as a hypointense feature on $\mathrm{T}_{1}$-weighted images in the arterial phase after which it becomes hyperintense in the equilibrium phase. One should also be aware of other hypervascular tumors. Fibrolamellar hepatocellular carcinoma (HCC) may, like $\mathrm{FNH}$, show a central scar that is more coarse and irregular (fig. 7). These scars 
Fig. 4. MRI with hepatobiliary contrast agent of a typical FNH. On the blank $\mathrm{T}_{1^{-}}$ weighted MRI, the lesion is visible, although isointense with a central scar (a). After contrast the lesion shows strong enhancement with exception of the central scar (b). In the portal venous phase, the lesion is slightly hyperintense with a hypointense central scar (c). In the hepatobiliary phase, the lesion is still hyperintense compared to the normal liver tissue (d).

Fig. 5. MRI with hepatobiliary contrast agent of a 'typical' HCA. MRI of multiple typical adenomas in a symptomless female patient. In the arterial phase, there is strong enhancement of the lesion (a). In the portal venous phase, the lesion is isointense (b). Due to rapid washout of contrast (washout phenomenon), the lesion becomes hypointense in the equilibrium phase (c). In the hepatobiliary phase, there is no enhancement of the hepatospecific contrast (d).
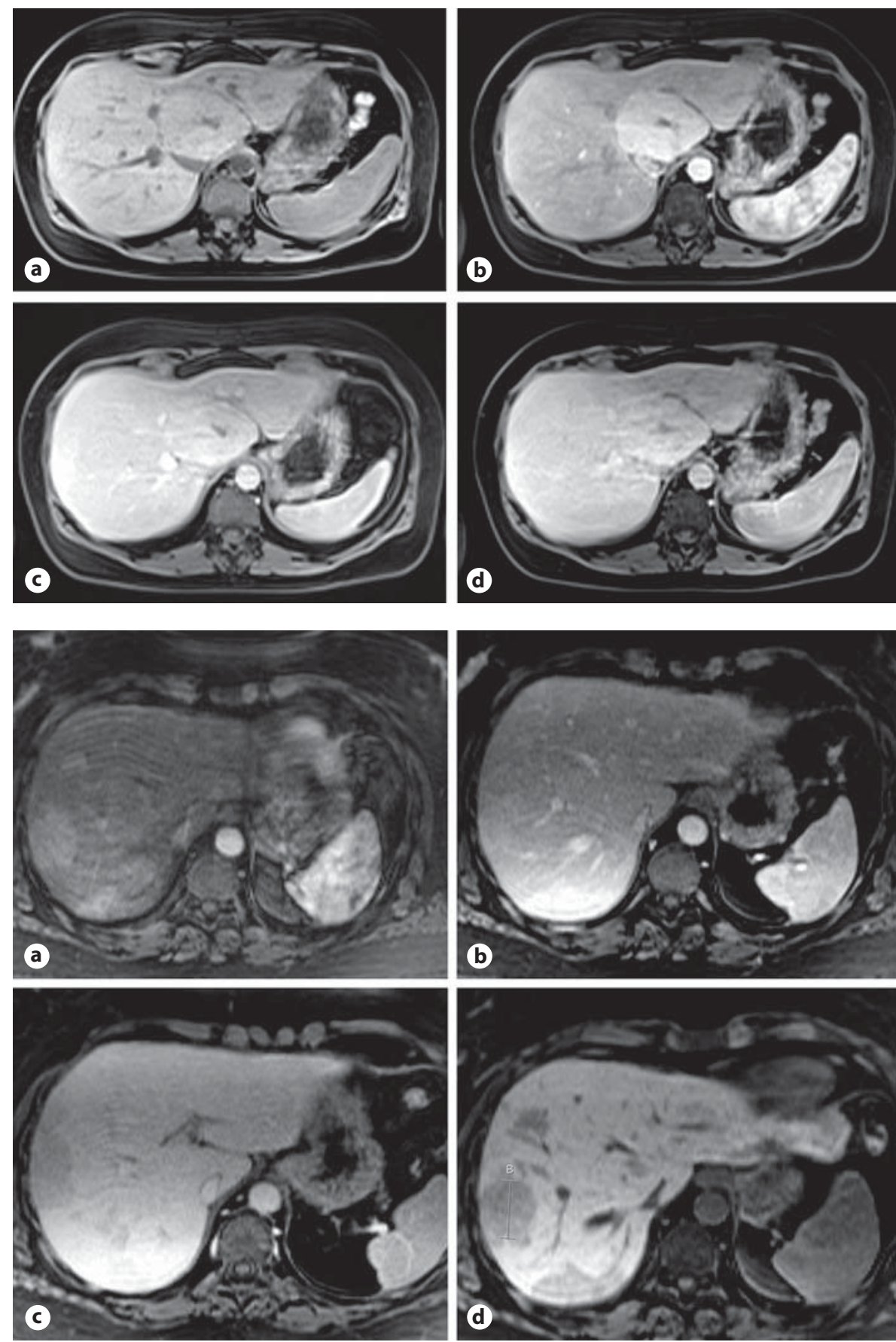

do not show late enhancement and the tumor often shows inhomogeneous enhancement and calcifications [29, 59]. Metastasis of renal cell carcinoma may also simulate FNH with a fibrotic central scar (fig. 8). These scars, however, do not show late enhancement. In HCA, an old central bleed may mimic a central scar. Heterogeneity, a peripheral rim and washout phenomena are common for both HCA and well-differentiated HCC (fig. 9). Furthermore, HCA and well-differentiated HCC occasionally show uptake of hepatocyte-specific contrast media, making a definitive diagnosis based on imaging alone impossible $[58,60,61]$. Metastasis from other hypervascular tumors (e.g. neuroendocrine tumors, carcinoid tumors and renal cell carcinoma) will, just as a typical HCA, not show 
Fig. 6. MRI with hepatobiliary contrast agent of atypical FNHs. On $\mathrm{T}_{1}$-weighted arterial phase images, two small lesions with strong homogeneous enhancement are visible (arrows) (a). During the hepatobiliary phase, only a slight ring-like enhancement is shown in the FNH (b).

Fig. 7. MRI with a lesion found in a 15 -yearold female with vague abdominal complaints. In the arterial phase, on $\mathrm{T}_{1}$-weighted imaging, strong enhancement of the lesion is seen except for the stellate central area (a). On the $\mathrm{T}_{2}$-weighted image, a low signal of the central 'scar' and coarse irregular aspect is clearly visible (b). The final diagnosis was a fibrolamellar HCC.
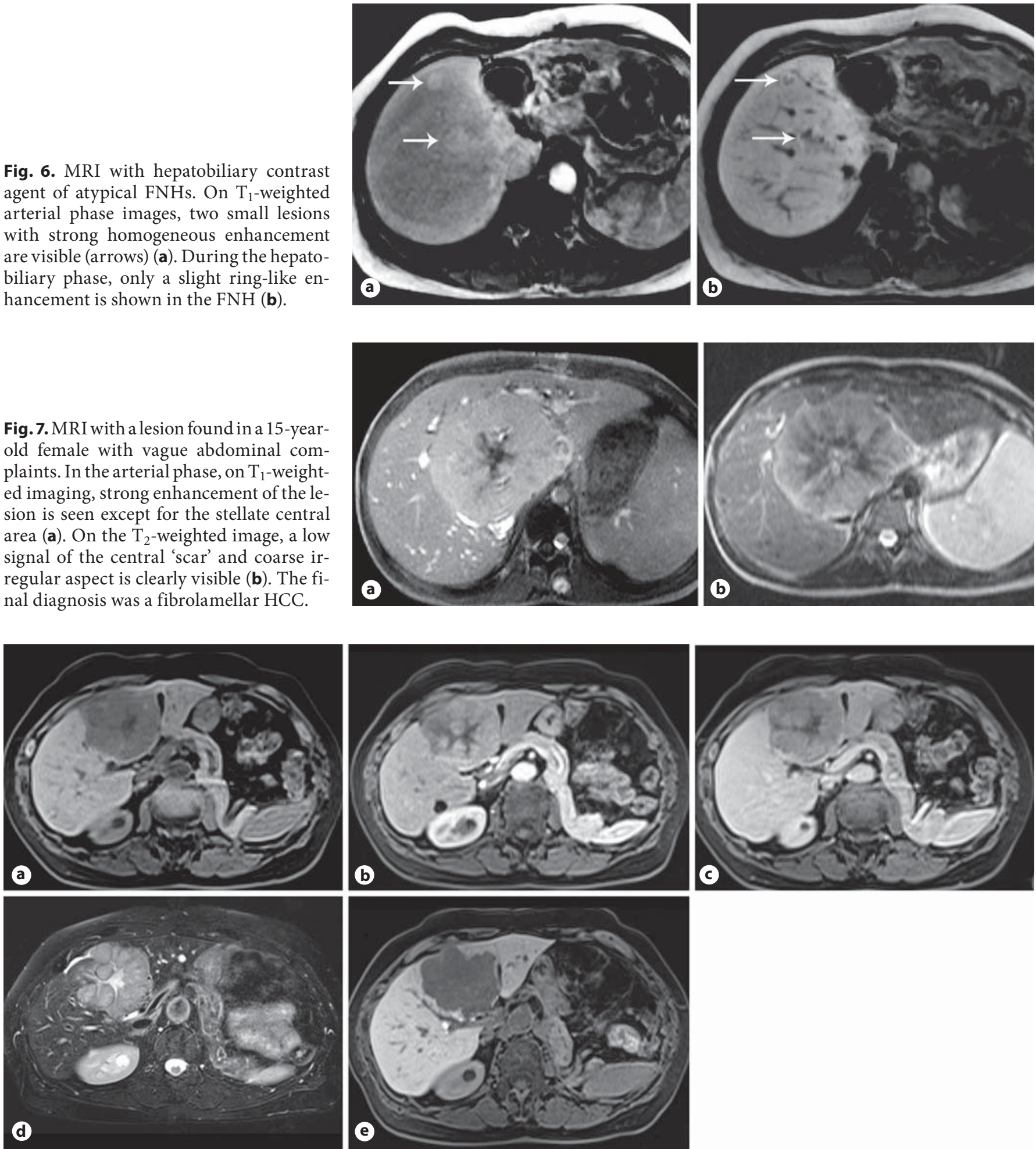

Fig. 8. MRI of a solitary lesion in a female patient. The lesion existed for 3 years and was considered an FNH. The lesion had increased in size. $\mathrm{T}_{1}$-weighted unenhanced MRI (a). After gadoxetic acid administration, there is strong enhancement of lesion with exception of the central stellate area in the arterial and portal

venous phase $(\mathbf{b}, \mathbf{c})$. On the $\mathrm{T}_{2}$-weighted image, a high signal of central 'scar' is visible (d). In the hepatobiliary phase, there is however no enhancement of contrast (e). The final diagnosis was a solitary metastasis of renal cell carcinoma, 14 years after resection of the primary tumor. 

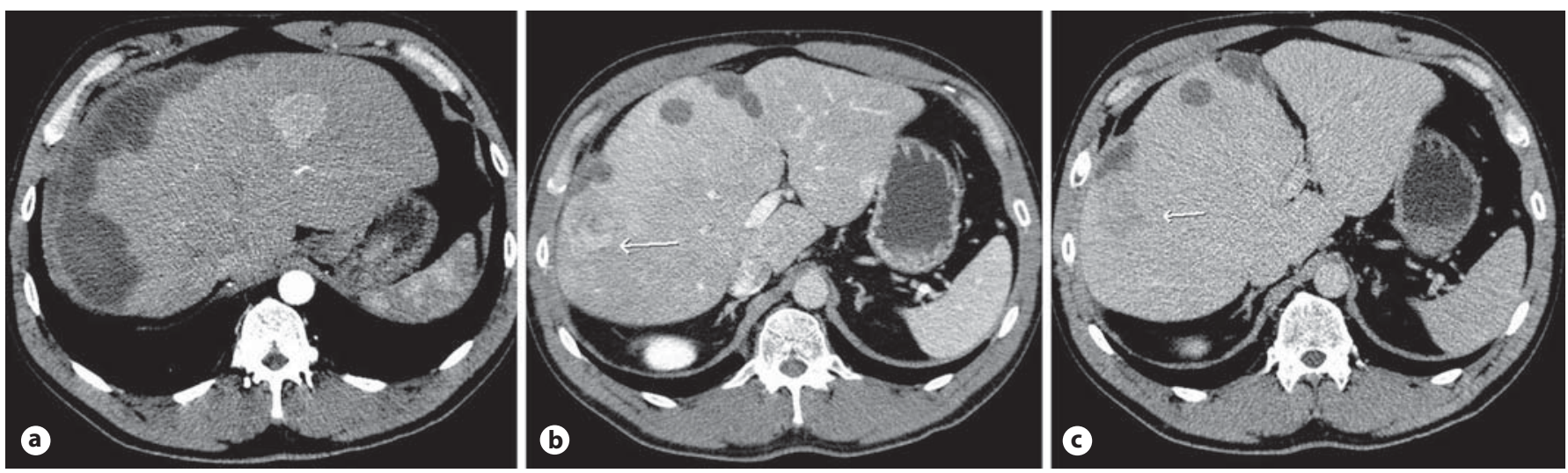

Fig. 9. CT scan of a male bodybuilder using steroids who presented with an acute bleeding. In the arterial phase, a bleeding and multiple enhancing lesions were seen (only one shown) (a). The portal venous and equilibrium phase images showed an inhomo- geneous lesion with a washout phenomenon (arrow) (b, c). This washout phenomenon was not found in the other lesions. The presumptive diagnosis was steroid-induced multiple adenomas, but the final diagnosis was HCC with metastases. uptake of hepatospecific MR contrast agents. Of note is a recent article in which a correlation is shown between features on MRI and the pathological subclassification of HCAs [62]. A detailed description of this study is however beyond the scope of this article.

\section{Nuclear Imaging}

On ${ }^{99 m}$ Tc-sulfur-colloid scintigraphy FNH usually shows normal or increased uptake, while HCA shows no uptake due to lack of functional Kupffer cells [63]. However, contrary to expectations, several studies showed that $30-36 \%$ of FNH have decreased uptake $[32,64,65]$. Herman et al. [66] used the ${ }^{99 \mathrm{~m}}$ Tc-sulfur-colloid scan preoperatively for the diagnosis of FNH $(\mathrm{n}=13)$ and HCA $(\mathrm{n}=10)$. Atypical findings were that 1 of the $13 \mathrm{FNH}$ showed a decreased uptake and 4 HCAs showed a normal uptake.

The hepatobiliary tracer ${ }^{99 \mathrm{~m}} \mathrm{Tc}-\mathrm{meb}$ rofenin (HIDA) is, like the hepatobiliary MR contrast agents, taken up by functional hepatocytes and excreted into the bile [64]. Since biliary drainage in FNH is reduced compared to normal liver parenchyma, the tracer is retained and visible as a hot spot on delayed images [65]. Evidence on the use of this imaging technique for the differentiation between FNH and HCA is however not available.

In 2007, Bumsel et al. [67] showed promising results in 14 patients in differentiating FNH from HCA using positron emission tomography (PET/CT) in combination with the use of the tracer fluoromethylcholine. So far, no series have however been published using this modality.

\section{Discussion}

Many imaging modalities are used to visualize FNH and HCA. Based on the typical features, especially FNH can be diagnosed with high certainty on several imaging studies. However, there are atypical findings in both FNH and HCA that preclude a definitive diagnosis and may resemble other tumors. While reviewing the literature, we found many case reports and studies which describe the features of FNH and HCA. However, there is little evidence on the diagnostic performance of imaging modalities in the differentiation of FNH and HCA. In many studies the final diagnosis was often only based on imaging and follow-up, while the gold standard is histopathological confirmation of the tumor. Moreover, the histopathological diagnosis of telangiectatic FNH has recently been reclassified as HCA on the basis of clinical and molecular features [68].

Bartolozzi et al. [13], Dietrich et al. [25] and Ruppert et al. [36] performed a performance study of Doppler-, contrast-enhanced US and multiphase helical CT scan, respectively, in patients with histology proven $\mathrm{FNH}$ or HCA. The sample sizes, especially for HCA, were however small. A large prospective, multicenter study conducted by Grazioli et al. [41] assessed the use of GdBOPTA MRI for the differentiation of FNH and HCA. They reported a sensitivity of $96.9 \%$ and a specificity of $100 \%$. However, a limitation of their study was that the gold standard, i.e. the histological diagnosis, was only available in less than $30 \%$ of the FNH lesions. The lack of histological confirmation of all lesions also applies to the 
retrospective study of Kim et al. [26]. No study compared the use of more than one imaging modality in the differentiation of FNH and HCA.

Because of the lack of reliable evidence, we started a prospective trial to assess the accuracy of several imaging studies for the differentiation of FNH and HCA, termed the DiFA trial which stands for the acronym 'differentiation of focal nodular hyperplasia and hepatocellular adenoma'. The aim of the DiFA trial is to compare the ac- curacy of a multiphase CT scan, MRI with Primovist ${ }^{\circledR}$, PET scan with fluoromethylcholine and contrast-enhanced US for the differentiation of FNH and HCA. The diagnosis based on the different imaging studies is compared with the histological outcome (core biopsy and/or resection specimen). The final aim is to develop a diagnostic algorithm for patients with suspicion of either $\mathrm{FNH}$ or HCA in order to obtain a correct diagnosis and provide proper treatment.

\section{References}

1 Huurman VAL, Schaapherder AFM: Management of ruptured hepatocellular adenoma. Dig Surg 2010, in press.

2 Farges O, Dokmak S: Malignant transformation of liver adenoma: an analysis of the literature. Dig Surg 2010, in press.

-3 Bioulac-Sage P, Balabaud C, Bedossa P, Scoazec JY, Chiche L, Dhillon AP, et al: Pathological diagnosis of liver cell adenoma and focal nodular hyperplasia: Bordeaux update. J Hepatol 2007;46:521-527.

4 Hussain SM, Terkivatan T, Zondervan PE, Lanjouw E, de RS, Ijzermans JN, et al: Focal nodular hyperplasia: findings at state-ofthe-art MR imaging, US, CT, and pathologic analysis. Radiographics 2004;24:3-17.

5 Vilgrain V: Focal nodular hyperplasia. Eur J Radiol 2006;58:236-245.

6 Buetow PC, Pantongrag-Brown L, Buck JL, Ros PR, Goodman ZD: Focal nodular hyperplasia of the liver: radiologic-pathologic correlation. Radiographics 1996;16:369-388.

7 Rogers JV, Mack LA, Freeny PC, Johnson ML, Sones PJ: Hepatic focal nodular hyperplasia: angiography, CT, sonography, and scintigraphy. AJR Am J Roentgenol 1981;137: 983-990.

8 Nguyen BN, Flejou JF, Terris B, Belghiti J, Degott C: Focal nodular hyperplasia of the liver: a comprehensive pathologic study of 305 lesions and recognition of new histologic forms. Am J Surg Pathol 1999;23:14411454.

9 Hung $\mathrm{CH}$, Changchien CS, Lu SN, Eng HL, Wang JH, Lee CM, et al: Sonographic features of hepatic adenomas with pathologic correlation. Abdom Imaging 2001;26:500506.

10 Grazioli L, Federle MP, Brancatelli G, Ichikawa T, Olivetti L, Blachar A: Hepatic adenomas: imaging and pathologic findings. Radiographics 2001;21:877-892.

-11 Wang LY, Wang JH, Lin ZY, Yu ML, Lu SN, Chuang WL, et al: Hepatic focal nodular hyperplasia: findings on color Doppler ultrasound. Abdom Imaging 1997;22:178-181.

12 Golli M, Mathieu D, Anglade MC, Cherqui D, Vasile N, Rahmouni A: Focal nodular hyperplasia of the liver: value of color Doppler
US in association with MR imaging. Radiology 1993;187:113-117.

-13 Bartolozzi C, Lencioni R, Paolicchi A, Moretti M, Armillotta N, Pinto F: Differentiation of hepatocellular adenoma and focal nodular hyperplasia of the liver: comparison of power Doppler imaging and conventional color Doppler sonography. Eur Radiol 1997; 7:1410-1415.

14 Burns PN, Wilson SR: Focal liver masses: enhancement patterns on contrast-enhanced images - concordance of US scans with CT scans and MR images. Radiology 2007;242: 162-174.

15 Bartolotta TV, Midiri M, Galia M, Rollandi GA, Cademartiri F, Lagalla R, et al: Characterization of benign hepatic tumors arising in fatty liver with SonoVue and pulse inversion US. Abdom Imaging 2007;32:84-91.

16 Ungermann L, Elias P, Zizka J, Ryska P, Klzo L: Focal nodular hyperplasia: spoke-wheel arterial pattern and other signs on dynamic contrast-enhanced ultrasonography. Eur J Radiol 2007;63:290-294.

17 Quaia E, Calliada F, Bertolotto M, Rossi S, Garioni L, Rosa L, et al: Characterization of focal liver lesions with contrast-specific US modes and a sulfur hexafluoride-filled microbubble contrast agent: diagnostic performance and confidence. Radiology 2004;232: $420-430$.

18 Li R, Guo Y, Hua X, He Y, Ding J, Guo A, et al: Characterization of focal liver lesions: comparison of pulse-inversion harmonic contrast-enhanced sonography with contrast-enhanced CT. J Clin Ultrasound 2007; 35:109-117.

19 Luo W, Numata K, Morimoto M, Nozaki A, Nagano Y, Sugimori K, et al: Three-dimensional contrast-enhanced sonography of vascular patterns of focal liver tumors: pilot study of visualization methods. AJR Am J Roentgenol 2009;192:165-173.

20 Xu HX, Liu GJ, Lu MD, Xie XY, Xu ZF, Zheng $Y L$, et al: Characterization of focal liver lesions using contrast-enhanced sonography with a low mechanical index mode and a sulfur hexafluoride-filled microbubble contrast agent. J Clin Ultrasound 2006;34:261-272.
21 Yen YH, Wang JH, Lu SN, Chen TY, Changchien CS, Chen $\mathrm{CH}$, et al: Contrastenhanced ultrasonographic spoke-wheel sign in hepatic focal nodular hyperplasia. Eur J Radiol 2006;60:439-444.

22 Brannigan M, Burns PN, Wilson SR: Blood flow patterns in focal liver lesions at microbubble-enhanced US. Radiographics 2004; 24:921-935.

23 Celli N, Gaiani S, Piscaglia F, Zironi G, Camaggi V, Leoni S, et al: Characterization of liver lesions by real-time contrast-enhanced ultrasonography. Eur J Gastroenterol Hepatol 2007;19:3-14.

24 Bartolotta TV, Taibbi A, Midiri M, Lagalla R: Focal liver lesions: contrast-enhanced ultrasound. Abdom Imaging 2009;34:193209.

25 Dietrich CF, Schuessler G, Trojan J, Fellbaum C, Ignee A: Differentiation of focal nodular hyperplasia and hepatocellular adenoma by contrast-enhanced ultrasound. $\mathrm{Br}$ J Radiol 2005;78:704-707.

-26 Kim TK, Jang HJ, Burns PN, Murphy-Lavallee J, Wilson SR: Focal nodular hyperplasia and hepatic adenoma: differentiation with low-mechanical-index contrast-enhanced sonography. AJR Am J Roentgenol 2008; 190: $58-66$.

-27 Brancatelli G, Federle MP, Grazioli L, Blachar A, Peterson MS, Thaete L: Focal nodular hyperplasia: CT findings with emphasis on multiphasic helical CT in 78 patients. Radiology 2001;219:61-68.

28 Carlson SK, Johnson CD, Bender CE, Welch TJ: CT of focal nodular hyperplasia of the liver. AJR Am J Roentgenol 2000;174:705712 .

29 Caseiro-Alves F, Zins M, Mahfouz A-E, Rahmouni A, Vilgrain V, Menu Y, et al: Calcification in focal nodular hyperplasia: a new problem for differentiation from fibrolamellar hepatocellular carcinoma. Radiology 1996;198:889-892.

30 Blachar A, Federle MP, Ferris JV, Lacomis JM, Waltz JS, Armfield DR, et al: Radiologists' performance in the diagnosis of liver tumors with central scars by using specific CT criteria. Radiology 2002;223:532-539. 
-31 Ichikawa T, Federle MP, Grazioli L, Nalesnik M: Hepatocellular adenoma: multiphasic CT and histopathologic findings in 25 patients. Radiology 2000;214:861-868.

- 32 Welch TJ, Sheedy PF, Johnson CM, Stephens DH, Charboneau JW, Brown ML, et al: Focal nodular hyperplasia and hepatic adenoma: comparison of angiography, CT, US, and scintigraphy. Radiology 1985; 156: 593-595.

-33 Kamel IR, Liapi E, Fishman EK: Focal nodular hyperplasia: lesion evaluation using 16MDCT and 3D CT angiography. AJR Am J Roentgenol 2006;186:1587-1596.

- 34 Mortele KJ, Praet M, Van VH, Kunnen M, Ros PR: CT and MR imaging findings in focal nodular hyperplasia of the liver: radiologic-pathologic correlation. AJR Am J Roentgenol 2000;175:687-692.

35 Choi BY, Nguyen MH: The diagnosis and management of benign hepatic tumors. J Clin Gastroenterol 2005;39:401-412.

- 36 Ruppert-Kohlmayr AJ, Uggowitzer MM, Kugler C, Zebedin D, Schaffler G, Ruppert GS: Focal nodular hyperplasia and hepatocellular adenoma of the liver: differentiation with multiphasic helical CT. AJR Am J Roentgenol 2001;176:1493-1498.

-37 Winterer JT, Kotter E, Ghanem N, Langer M: Detection and characterization of benign focal liver lesions with multislice CT. Eur Radiol 2006; 16:2427-2443.

- 38 Lin MC, Tsay PK, Ko SF, Lui KW, Tseng JH, Hung CF, et al: Triphasic dynamic CT findings of 63 hepatic focal nodular hyperplasia in 46 patients: correlation with size and pathological findings. Abdom Imaging 2008;33: 301-307.

-39 Grandin C, van Beers BE, Robert A, Gigot JF, Geubel A, Pringot J: Benign hepatocellular tumors: MRI after superparamagnetic iron oxide administration. J Comput Assist Tomogr 1995; 19:412-418.

-40 Grazioli L, Morana G, Federle MP, Brancatelli G, Testoni M, Kirchin MA, et al: Focal nodular hyperplasia: morphologic and functional information from MR imaging with gadobenate dimeglumine. Radiology 2001; 221:731-739.

-41 Grazioli L, Morana G, Kirchin MA, Schneider G: Accurate differentiation of focal nodular hyperplasia from hepatic adenoma at gadobenate dimeglumine-enhanced MR imaging: prospective study. Radiology 2005;236:166-177.

-42 Mathieu D, Rahmouni A, Anglade MC, Falise B, Beges C, Gheung P, et al: Focal nodular hyperplasia of the liver: assessment with contrast-enhanced TurboFLASH MR imaging. Radiology 1991;180:25-30.

-43 Dill-Macky M, Frazer C, de Boer WB: Magnetic resonance features of focal nodular hyperplasia of the liver. Australas Radiol 1999; 43:315-320.

-44 Mahfouz AE, Hamm B, Taupitz M, Wolf KJ: Hypervascular liver lesions: differentiation of focal nodular hyperplasia from malignant tumors with dynamic gadolinium-enhanced MR imaging. Radiology 1993;186:133-138.

45 Mattison GR, Glazer GM, Quint LE, Francis IR, Bree RL, Ensminger WD: MR imaging of hepatic focal nodular hyperplasia: characterization and distinction from primary malignant hepatic tumors. AJR Am J Roentgenol 1987;148:711-715.

46 Mortele KJ, Praet M, Van VH, de HB, Zou K, Ros PR: Focal nodular hyperplasia of the liver: detection and characterization with plain and dynamic-enhanced MRI. Abdom Imaging 2002;27:700-707.

-47 Terkivatan T, van den Bos IC, Hussain SM, Wielopolski PA, de Man RA, IJzermans JN: Focal nodular hyperplasia: lesion characteristics on state-of-the-art MRI including dynamic gadolinium-enhanced and superparamagnetic iron-oxide-uptake sequences in a prospective study. J Magn Reson Imaging 2006;24:864-872.

$\checkmark 48$ Zech CJ, Grazioli L, Breuer J, Reiser MF, Schoenberg SO: Diagnostic performance and description of morphological features of focal nodular hyperplasia in Gd-EOBDTPA-enhanced liver magnetic resonance imaging: results of a multicenter trial. Invest Radiol 2008;43:504-511.

49 Chung KY, Mayo-Smith WW, Saini S, Rahmouni A, Golli M, Mathieu D: Hepatocellular adenoma: MR imaging features with pathologic correlation. AJR Am J Roentgenol 1995; 165:303-308.

50 Paulson EK, McClellan JS, Washington K, Spritzer CE, Meyers WC, Baker ME: Hepatic adenoma: MR characteristics and correlation with pathologic findings. AJR Am J Roentgenol 1994;163:113-116.

51 Arrive L, Flejou JF, Vilgrain V, Belghiti J, Najmark D, Zins M, et al: Hepatic adenoma: MR findings in 51 pathologically proved lesions. Radiology 1994;193:507-512.

52 Scharitzer M, Schima W, Schober E, Reimer P, Helmberger TK, Holzknecht N, et al: Characterization of hepatocellular tumors: value of mangafodipir-enhanced magnetic resonance imaging. J Comput Assist Tomogr 2005;29:181-190.

53 Denys A, Arrive L, Servois V, Dubray B, Najmark D, Sibert A, et al: Hepatic tumors: detection and characterization at 1-T MR imaging enhanced with AMI-25. Radiology 1994;193:665-669.

-54 Precetti-Morel S, Bellin MF, Ghebontni L, Zaim S, Opolon P, Poynard T, et al: Focal nodular hyperplasia of the liver on ferumoxides-enhanced MR imaging: features on conventional spin-echo, fast spin-echo and gradient-echo pulse sequences. Eur Radiol 1999;9:1535-1542.

55 Paley MR, Mergo PJ, Torres GM, Ros PR: Characterization of focal hepatic lesions with ferumoxides-enhanced $\mathrm{T}_{2}$-weighted MR imaging. AJR Am J Roentgenol 2000; 175:159-163.

56 Beets-Tan RG, Van Engelshoven JM, Greve JW: Hepatic adenoma and focal nodular hy- perplasia: MR findings with superparamagnetic iron oxide-enhanced MRI. Clin Imaging 1998;22:211-215.

57 Hamm B, Vogl TJ, Branding G, Schnell B, Taupitz M, Wolf KJ, et al: Focal liver lesions: MR imaging with Mn-DPDP-initial clinical results in 40 patients. Radiology 1992;182: 167-174.

58 Huppertz A, Haraida S, Kraus A, Zech CJ, Scheidler J, Breuer J, et al: Enhancement of focal liver lesions at gadoxetic acid-enhanced MR imaging: correlation with histopathologic findings and spiral CT - initial observations. Radiology 2005;234:468-478.

59 Hamrick-Turner JE, Shipkey FH, Cranston PE: Fibrolamellar hepatocellular carcinoma: MR appearance mimicking focal nodular hyperplasia. J Comput Assist Tomogr 1994; 18:301-304.

60 Ishigami K, Yoshimitsu K, Nishihara Y, Irie $\mathrm{H}$, Asayama Y, Tajima T, et al: Hepatocellular carcinoma with a pseudocapsule on gadolinium-enhanced MR images: correlation with histopathologic findings. Radiology 2009; 250:435-443.

61 Halavaara J, Breuer J, Ayuso C, Balzer T, Bellin MF, Blomqvist L, et al: Liver tumor characterization: comparison between liverspecific gadoxetic acid disodium-enhanced MRI and biphasic CT - a multicenter trial. J Comput Assist Tomogr 2006;30:345-354.

62 Laumonier H, Bioulac-Sage P, Laurent C, Zucman-Rossi J, Balabaud C, Trillaud H: Hepatocellular adenomas: magnetic resonance imaging features as a function of molecular pathological classification. Hepatology 2008;48:808-818.

63 Casarella WJ, Knowles DM, Wolff M, Johnson PM: Focal nodular hyperplasia and liver cell adenoma: radiologic and pathologic differentiation. AJR Am J Roentgenol 1978;131: 393-402.

64 Biersack HJ, Thelen M, Torres JF, Lackner K, Winkler CG: Focal nodular hyperplasia of the liver as established by ${ }^{99 \mathrm{~m}} \mathrm{Tc}$ sulfur colloid and HIDA scintigraphy. Radiology 1980;137: 187-190.

65 Boulahdour H, Cherqui D, Charlotte F, Rahmouni A, Dhumeaux D, Zafrani ES, et al: The hot spot hepatobiliary scan in focal nodular hyperplasia. J Nucl Med 1993;34:2105-2110.

-66 Herman P, Pugliese V, Machado MA, Montagnini AL, Salem MZ, Bacchella T, et al: Hepatic adenoma and focal nodular hyperplasia: differential diagnosis and treatment. World J Surg 2000;24:372-376.

67 Bumsel F, Huchet V, Arrive L, Wendum D, Paye F, Poupon R, et al: Positron emission tomography (PET/CT) using fluorocholine allows to differentiate between adenoma and focal nodular hyperplasia. Hepatology 2007; 468(suppl 1).

68 Bioulac-Sage P, Rebouissou S, Thomas C, Blanc JF, Saric J, Sa CA, et al: Hepatocellular adenoma subtype classification using molecular markers and immunohistochemistry. Hepatology 2007;46:740-748. 